\title{
MONITORING AGRICULTURAL FIELDS USING AN OPTIMISATION OF THE DIFFERENCE OF COVARIANCE MATRICES FOR POLSAR
}

\author{
Cristian Silva $^{1}$, Armando Marino ${ }^{1}$, Juan M. Lopez-Sanchez ${ }^{2}$ and Iain Cameron ${ }^{3}$ \\ ${ }^{1}$ The Open University, Engineering and Innovation, Milton Keynes, United Kingdom \\ ${ }^{2}$ University of Alicante, IUII, Alicante, Spain \\ ${ }^{3}$ Environment systems Ltd, Aberystwyth, United Kingdom
}

\begin{abstract}
SAR polarimetry (PolSAR) can play an important role in monitoring agricultural fields both in terms of improving detection of specific plants conditions and providing physical information regarding the change. Such information can be used to help retrieving the phenological stage and eventually identifying stress conditions.

In this work, a new change detection based on PolSAR data is first tested over time series of images acquired over agricultural fields. The methodology is based on the use of the normalised difference between covariance matrices acquired at two different instants. A diagonalisation of such matrix allows identifying the scattering mechanisms that suffer the largest change. The methodology is tested exploiting C-band quad-polarimetric RADARSAT-2 data over rice fields in Sevilla, South-West of Spain.
\end{abstract}

Index Terms- Agriculture, polarimetry, change detection, SAR.

\section{INTRODUCTION}

In the following, a very brief introduction to PolSAR is proposed with the only purpose of presenting the mathematical formalism exploited in this work. A single target has a fixed polarisation in time/space and we can characterise it using the scattering (Sinclair) matrix or equivalently a scattering vector $\underline{k}$ [1]. It is possible to define a projection vector as a normalised vector $\underline{\omega}=\frac{k}{\|k\|}$. The target observed by a SAR system is not an idealised scattering target, but a combination of different targets which we refer to as a partial target. In order to characterise a partial target the single scattering matrix is not sufficient since it is a stochastic process, and the second order statistics are required. In this context the target covariance matrix can be estimated: $C=\left\langle\underline{k} \underline{k}^{* T}\right\rangle$, where $*$ stands for conjugate, $T$ for transpose, and $\langle$.$\rangle is the finite averaging$ operator [1].

In this work, we use the sample coherency matrix $T$, that is a covariance matrix formed after applying a Pauli decom- position to the scattering vector $\underline{k}_{p}=\frac{1}{\sqrt{2}}[H H+V V, H H-$ $V V, 2 H V]^{T}$ ).

Polarimetry can help improving the capability of change detectors and there are several works in the literature focused on this $[2,3,4,5]$.

\subsection{Signal Model}

The two acquisitions to be compared are referred to as date1 and date 2 , so $T_{11}$ and $T_{22}$ are the coherency matrices characterising the partial targets at datel and date2, respectively.

The change detector developed in this work is based on a signal model that considers independence between the scattering mechanism present at the first acquisition and the change. The hypothesis of independence of scattering mechanisms is largely exploited for change detection. For instance a wellknown algorithm, Wishart change detector [4], is based on this concept.

In this work, the independence hypothesis is relaxed since the partial targets represented by $T_{11}$ and $T_{22}$ are not required to be independent. This means that we do not make any hypothesis on the correlation between $\underline{k}_{1}$ and $\underline{k}_{2}$, which can assume any value. This entails that the Pol-InSAR matrix $\left[\Omega_{12}\right]$ (which evaluates the outer product between $\underline{k}_{1}$ and $\underline{k}_{2}$ ) has not to be a null matrix, and the algorithm is compatible with other Pol-InSAR methodologies.

Since it is only the change that is independent of $T_{11}$, we can write $T_{22}$ as the sum of $T_{11}$ plus an independent scattering mechanism $T_{c}$ :

$$
T_{22}=T_{11}+T_{c},
$$

where $T_{c}$ is defined as "change matrix" and it is generated by a process that is independent of the process generating $T_{11}$ (but it is not independent of the process generating $T_{22}$ ). Therefore the processes generating $T_{11}$ and $T_{22}$ are not independent of each other.

\subsection{Normalised difference}

The change matrix $T_{c}$ can be calculated as $T_{c}=T_{22}-T_{11}$. $T_{c}$ is Hermitian, since it is the difference of two Hermitian 
matrices. This means that the diagonal elements of $T_{c}$ are real and the upper triangular part is the complex conjugate of the lower triangular part. However, there is a difference with ordinary coherencies matrix, since it is not bound to be positive semi-definite. This means that its quadratic forms (using a generic projection vector) or its Trace can be negative. This means that $P=\underline{\omega}^{* T} T_{c} \underline{\omega}$ can be negative for some projection vector $\underline{\omega}$, and $\operatorname{Trace}\left(\left[T_{c}\right]\right)$ can be negative.

This is an expected feature since we need a matrix that is able to communicate if the change in the partial target has brought an increase or reduction of either a) a specific scattering mechanism (quadratic form), or b) the overall power of the final partial target (trace).

By optimising $T_{c}$ over all the possible projection vectors $\underline{\omega}$ it is possible to derive the projection vectors that experience the largest or smallest changes.

In this work we decided to normalise the difference since this allows us to evaluate the changes weighted on the total backscattering coming from the partial targets. This is convenient to avoid that very bright targets may return large differences even when they are experiencing very small changes (in percentage). The normalisation can be accomplished using the total power of both partial targets (i.e. the Trace).

Therefore, the final matrix that is considered in the optimisation is:

$$
T_{C}=\frac{T_{22}-T_{11}}{\operatorname{Trace}\left(T_{11}\right)+\operatorname{Trace}\left(T_{22}\right)}
$$

The quadratic form $\underline{\omega}^{* T} T_{C} \underline{\omega}$ returns the amount of change that the scattering vector represented by the projection vector $\omega$ suffers. If we adopt the previously stated signal model, $\omega$ also provides the scattering mechanism that was added/subtracted in date 2 to the partial target in datel.

\subsection{Optimisation}

To find the maximum and minimum projection vectors we can apply the well-known Lagrangian optimisation for the quadratic form $\underline{\omega}^{* T} T_{C} \underline{\omega}$. That is:

$$
\underline{\omega}_{\max }=\underset{\underline{\omega} \in \mathbb{C}^{3}}{\operatorname{Argmax}}\left[\underline{\omega}^{* T} T_{C} \underline{\omega}\right] .
$$

By constraining $\omega$ to be unitary we can obtain the Lagrangian as:

$$
L=\underline{\omega}^{* T} T_{C} \underline{\omega}-\lambda\left(\underline{\omega}^{* T} \underline{\omega}+C\right) .
$$

After differentiating over $d \underline{\omega}^{* T}$ and setting the derivative equal to zero we obtain the equation:

$$
T_{C} \underline{\omega}=\lambda \underline{\omega} .
$$

The Lagrangian multipliers can be calculated by performing a diagonalisation of the matrix $T_{C}$. Since $T_{C}$ is Hermitian, the eigenvalues will be real, but not necessarily positive.
This is because a change can increase or decrease the resulting power of a scattering mechanism (e.g. we could have that surface scattering increases or decreases).

This optimisation wants also to complement one developed by the authors $[6,7]$ that was aimed at optimising the power ratio. The eigenvalues and eigenvectors of these two algorithms are different and could be used in a complementary way. Future work will be dedicated to this.

\subsection{Classifying the changes}

The outputs of the optimisation are the eigenvalues $\lambda_{i}$ and the eigenvectors $\underline{u}_{i}$ (with i going from 1 to 3 ) of $T_{C}$.

Interestingly, with this signal model, the eigenvectors $\underline{u}_{i}$ represent directly the scattering mechanisms that exhibit the changes. This vector can therefore be used to classify the type of change that the target is suffering. The way the eigenvectors are represented also plays an important role when coming to visualising the results. In our case, we used a decomposition which is mathematically equivalent to the Cloude-Pottier decomposition. This means that each eigenvector can be characterised using polar coordinates, and the $\alpha$ and $\beta$ angles are extracted. The $\alpha$ angle characterises the type of scattering (e.g. surface, dipole, dihedral) whereas the $\beta$ angle is proportional to the target orientation [1].

When the $\alpha$ angle is applied to the eigenvectors of the change detector, it identifies the dominant scattering mechanism that has been added to the partial target and the one that has been removed. For this reason, it should be linked to physical targets that are introduced during the development d of the plants. A possible application of this classification is for instance monitoring an increase in dihedral scattering due to the process of emerging of the plant stems. The transition between different phenological stages should be represented by the introduction or removal of different scattering mechanisms. Therefore, analysing the trend of the eigenvectors we aim at identifying the transition between successive phenological stages.

\section{RESULTS WITH REAL DATA}

\subsection{Presentation of the data}

The algorithm was tested on C-band RADARSAT-2 data acquired in spring and summer 2014 over rice fields in Sevilla, South-West of Spain. The data are quad-polarimetric. In this test, five images going across the entire cultivation cycle of rice are selected. The acquisition dates are 2014/06/05, 2014/06/29, 2014/07/23, 2014/08/16 and 2014/09/09. The Pauli RGB image corresponding to August is presented for reference in the Figure 1, including a region of interest where the phenology of the plants in a parcel is known. 


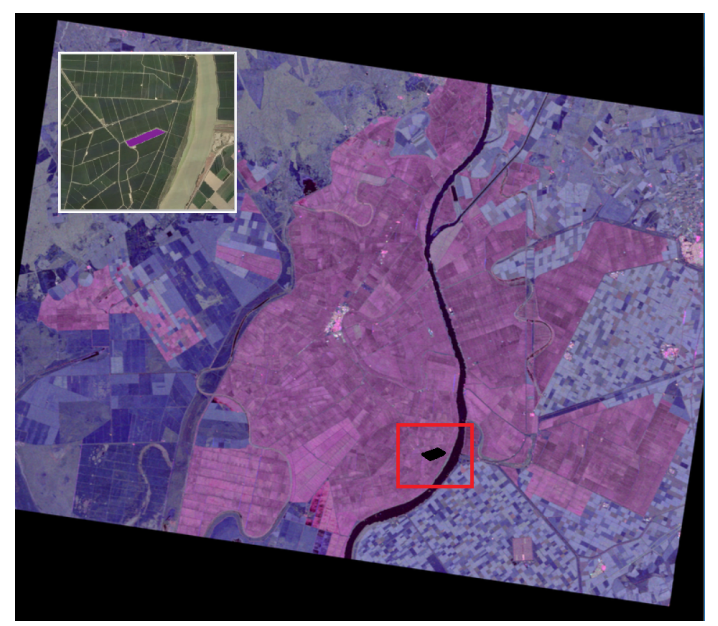

Fig. 1. Pauli RGB composite of the rice fields in Sevilla, Spain. RADARSAT-2 quad-pol data of 2014/08/16

\subsection{Detector}

The maximum eigenvalues represent the intensity of the scattering mechanism that has been added and removed to the previous partial target. These are presented by (a) and (b) in the Figures 2 to 5. The intensity of the scattering mechanisms presents the biggest increases in the three initial acquisitions, that is, at the beginning of the cultivation campaign.

According to the ground truth in the known parcel, these dates correspond to vegetative stage of the plants from $\mathrm{BBCH}$ 10 to $\mathrm{BBCH} 35$ with the measurements based on the $\mathrm{BBCH}$ scale as explained in $[8,9])$. Here, more vegetative structures are added to the scattering scene due to the increase in the plants height and the number of tillers. As a consequence, there is an increase in the intensity of the scattering mechanisms.

On the contrary, the biggest decrease in the intensity occurs in the transition between vegetative to reproductive stages of the plant as obtained by the change detector between the third and fourth acquisitions (figure 4.b). This is observed due to the the lower backscattered power at VV polarization caused by the extinction effect because of the vertical orientation of the plants as has been reported in $[8,9]$.

The intensity change in the last couple of acquisitions, from the reproductive to the maturation stages of the plants, indicates simultaneous increase and decrease of the scattering mechanisms present in the scene (figure $5 \mathrm{a}$ and $\mathrm{b}$ ). This is consequence of the plants random orientation and can be better interpreted with the aid of the alpha angle.

Looking at the $\alpha$ angles we can observe that the main mechanism that seem to be added in the early vegetative state is dihedral (figure 2.c). This is assumed to be produced by the double bounce formed by the plant stems and the water surface under the plants during the tillering stage. This process seems to happen at slightly different times for different fields and therefore such scattering mechanism could be an indicator of the emerging of stems outside the water.

The advanced vegetative stage shows an increase in dipole scattering (figure 3.c), which could be related to the formation of larger stems which may hide the water surface under the plants. In the maturation stage, there is a slight increase in surface and dipole scattering (figure 5.c), and a decrease in dihedral scattering (figure 5.d) that could be due to plants getting drier and therefore more transparent to the radiation allowing to reach the ground.

\section{CONCLUSIONS}

In this study RADARSAT-2 quad-polarimetric data were used to monitor rice fields in the South of Spain. The optimisation of the normalised difference of coherency matrices was used to track the physical changes of the rice fields. Such detector is able to identify the dominant scattering mechanisms that are added to or removed from the previous partial targets.

Specifically, in the early vegetative stage we have the addition of dihedral scattering due to emerging stems. Following this, there is an addition of dipoles during the reproductive stage and finally a combination of surface scattering and dipoles, and reduction of dihedral scattering.

\section{ACKNOWLEDGEMENT}

This research was partially supported by a mobility Scholarship from the Santander Bank, UK, and the Project EO4cultivar, led by Environment Systems Ltd and co-funded by the UK Space Agency.

This work was partially supported by the Spanish Ministry of Economy, Industry and Competitiveness (MINECO), the State Agency of Research (AEI) and the European Funds for Regional Development (EFRD) under Project TEC201785244-C2-1-P.

RADARSAT-2 Data and Products (C) MacDonald, Dettwiler and Associates Ltd. (2014) - All Rights Reserved. RADARSAT is an official trademark of the Canadian Space Agency. The data were provided under the SOAR Education International Initiative (SOAR-EI-5158).

\section{REFERENCES}

[1] S. R. Cloude, Polarisation: Applications in Remote Sensing, Oxford University Press, Oxford, UK, 2009.

[2] A. Alonso-Gonzalez, C. Lopez-Marinez, and P. Salembier, "PolSAR time series processing with binary partition trees," vol. 52, no. 6, pp. 3553-3567, 2014.

[3] V. Carotenuto, A. De Maio, C. Clemente, and J. J. Soraghan, "Invariant rules for multipolarization SAR change detection.," IEEE Transactions on Geoscience and Remote Sensing, vol. 53, no. 6, pp. 3294-3311, June 2015. 


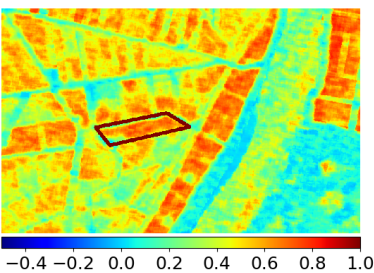

(a)Increase in intensity

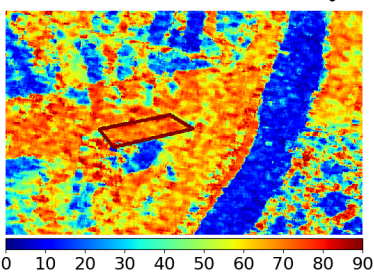

(c)Added Scattering mech.

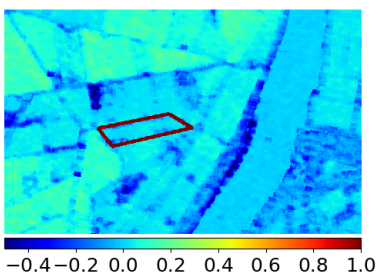

(b)Decrease in intensity

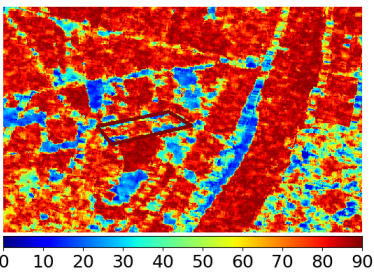

(d)Removed Scattering mech.
Fig. 2. Change detection between 2014/06/05 and 2014/06/29 of rice fields in Sevilla, Spain. The increase/decrease in intensity is measured between -0.5 and 1 . The $\alpha$ angle is scaled between 0 and $\frac{\pi}{2}$ degrees with the same interpretation as the Cloude-Pottier decomposition. The addition or reduction of a scattering mechanism is proportional to the intensity determined by the respective eigenvalue.

[4] K. Conradsen, A. A. Nielsen, J. Schou, and H. Skriver, "A test statistic in the complex Wishart distribution and its application to change detection in polarimetric SAR data," IEEE Trans. on Geos. \& Rem. Sen., vol. 41, 2003.

[5] A. Marino, S. R. Cloude, and J. M. Lopez-Sanchez, "A new polarimetric change detector in radar imagery," IEEE Transactions on Gescience and Remote Sensing, vol. 51, no. 5, pp. $2986-3000,2013$.

[6] A. Marino and I. Hajnsek, "A change detector based on an optimization with polarimetric SAR imagery," IEEE Transactions on Geoscience and Remote Sensing, vol. 52, no. 8, pp. 4781-4798, Aug. 2014.

[7] A. Alonso-Gonzlez, K. Papathanassiou, and I. Hajnsek, "Physical interpretation of the polarimetric SAR changes observed over agricultural time series.," 2015.

[8] J. M. Lopez-Sanchez, F. Vicente-Guijalba, J. D. Ballester-Berman, and S. R. Cloude, "Polarimetric response of rice fields at C-band: Analysis and phenology retrieval," IEEE Trans. on Geos. \& Rem. Sen., vol. 52, no. $5,2014$.

[9] J. M. Lopez-Sanchez, F. Vicente-Guijalba, E. Erten, M. Campos-Taberner, and F. Javier Garcia-Haro, "Retrieval of vegetation height in rice fields using polarimetric SAR interferometry with TanDEM-X data," Remote Sensing of Environment, vol. 192, pp. 30-44, 2017.

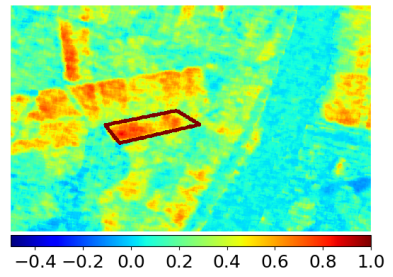

(a)Increase in intensity

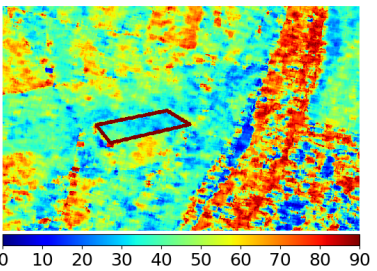

(c)Added Scattering mech.

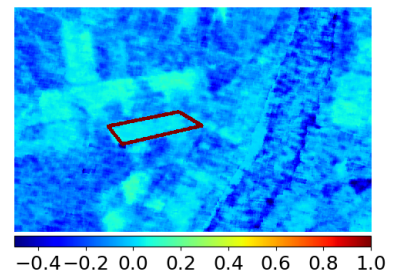

(b)Decrease in intensity

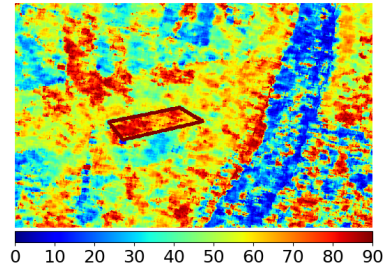

(d)Removed Scattering mech.
Fig. 3. Change detection between 2014/06/29 and 2014/07/23

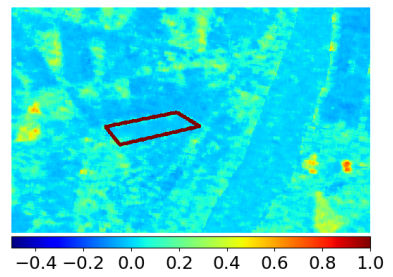

(a)Increase in intensity

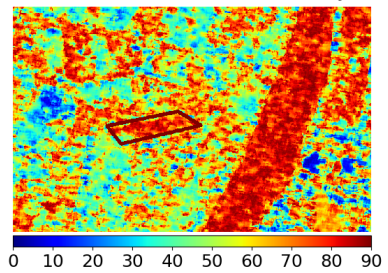

(c)Added Scattering mech.

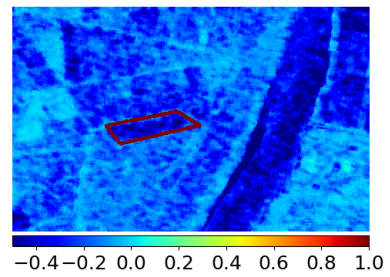

(b)Decrease in intensity

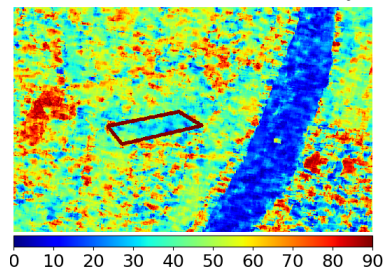

(d)Removed Scattering mech.
Fig. 4. Change detection between 2014/07/23 and 2014/08/16

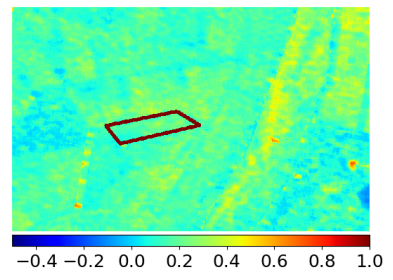

(a)Increase in intensity

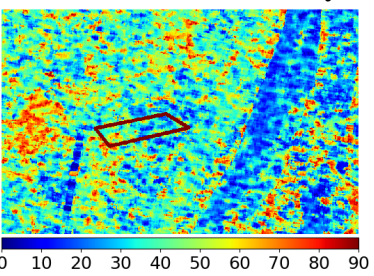

(c)Added Scattering mech.

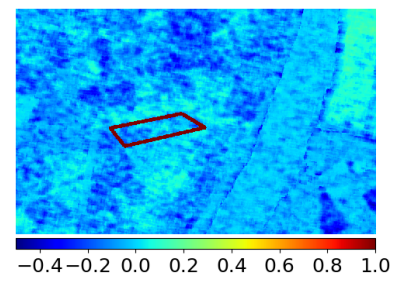

(b)Decrease in intensity

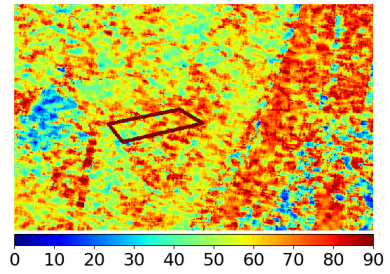

(d)Removed Scattering mech.
Fig. 5. Change detection between 2014/08/16 and 2014/09/09 